\title{
Intermédialités
}

Histoire et théorie des arts, des lettres et des techniques

Intermediality

History and Theory of the Arts, Literature and Technologies

\section{La migration de l'aura ou comment explorer un original par le biais de ses fac-similés}

\section{Bruno Latour et Adam Lowe}

Numéro 17, printemps 2011

URI : https://id.erudit.org/iderudit/1005756ar

DOI : https://doi.org/10.7202/1005756ar

Aller au sommaire du numéro

Éditeur(s)

Revue intermédialités (Presses de l’Université de Montréal)

ISSN

1705-8546 (imprimé)

1920-3136 (numérique)

Découvrir la revue

Citer cet article

Latour, B. \& Lowe, A. (2011). La migration de l'aura ou comment explorer un original par le biais de ses fac-similés. Intermédialités / Intermediality, (17), 173-191. https://doi.org/10.7202/1005756ar
Résumé de l'article

En prenant l'exemple de la duplication par l'intermédiaire de techniques numériques des Noces de Cana de Véronèse et de son accrochage dans son lieu palladien d'origine à San Giorgio à Venise, les auteurs envisagent la possibilité d'un détachement de l'aura par rapport à l'oeuvre originale. Grâce aux nouvelles techniques de fac-similisation, l'aura pourrait s'attacher à l'une ou l'autre des reproductions selon la qualité de ce que l'on peut considérer être les versions d'une oeuvre et selon la réussite de leur inscription dans un lieu particulier. Selon cette nouvelle logique, parallèle à celle des arts performatifs, l'oeuvre originale ne serait plus qu'une matrice première, l'origine d'une longue lignée de reproductions qui viendrait l'actualiser. Et dans la mesure où tous les originaux devraient être reproduits pour " survivre ", il importera désormais de savoir distinguer les bonnes des mauvaises reproductions. 


\title{
La migration de l'aura ou comment explorer un original par le biais de ses fac-similés*
}

\author{
Bruno latour, Adam Lowe
}

À Pasquale Gagliardi

\begin{abstract}
Q uelque chose d'étrange est arrivé aux Ambassadeurs de Holbein à la National Gallery de Londres. Au premier coup d'œil, la visiteuse ne sait pas trop comment décrire son malaise. Le tableau est complètement aplani, ses couleurs sont claires, mais quelque peu criardes. La visiteuse peut toujours distinguer la forme de chacun des objets; toutefois, ceux-ci paraissent légèrement exagérés. Elle se demande ce qui a bien pu arriver à son tableau préféré. "Voilà!, murmure-t-elle, le tableau a perdu de sa profondeur, la dynamique fluide du pigment n'y est plus. Il ne s'agit à présent que d'une surface. » Or, à quoi cette surface ressemble-t-elle? La visiteuse regarde autour d'elle, perplexe; d'un coup, une réponse surgit: cette surface est presque identique à l'affiche qu'elle s'est procurée il y a quelques années à la librairie du musée, celle qui est toujours accrochée chez elle dans son bureau. La seule différence tient aux dimensions.

Est-ce vrai? Elle réfléchit. Pourraient-ils avoir remplacé les Ambassadeurs par un fac-similé? Peut-être que le tableau a été prêté à d'autres musées et, afin de ne pas décevoir les visiteurs, ils se seraient contentés d'accrocher cette copie. Ou peut-être qu'ils ne souhaitaient pas nous leurrer et qu'il s'agit, après tout, d'une projection. L'image est tellement aplatie et elle est si vive qu'il pourrait bien s'agir d'une diapositive projetée sur un écran, ou presque... Heureusement, la visiteuse se ressaisit, évitant ainsi de s'enquérir auprès du gardien austère afin de

* Nous remercions les participants du Dialogue tenu à San Giorgio à Venise sur le thème "Inheriting the Past» pour les nombreux et utiles échanges et, spécialement, le directeur de la Fondation Cini, Pasquale Gagliardi.
\end{abstract}


savoir s'il s'agit de l'original de ce célébrissime tableau. Quel choc cela lui aurait causé. Malheureusement, elle en sait suffisamment sur les coutumes étranges des restaurateurs et des conservateurs pour se rendre à l'évidence que d'original, il ne conserve plus que le titre. Dès lors, elle constate que l'original a été irrévocablement perdu et que ce qui s'y est substitué possède les principales caractéristiques que la plupart des gens recherchent dans des copies: couleurs vives, surface scintillante et, surtout, le fait de ressembler parfaitement aux diapositives qui sont vendues à la librairie et qui sont projetées dans des cours d'histoire de l'art partout dans le monde par des enseignants s'intéressant surtout aux formes et aux thèmes des tableaux, et non pas aux marques inscrites sur la surface des œuvres et attestant de leur matérialité. Elle quitte la salle en retenant une larme: l'original a été transformé en une copie de lui-même ressemblant à une copie de mauvaise qualité - et personne ne semble se plaindre ou même se rendre compte de la substitution. Ils sont tous contents d'avoir rendu visite à l'affiche originale des Ambassadeurs de Holbein se trouvant à Londres!

Quelque chose de plus étrange encore est arrivé à notre visiteuse dans la salle de la Joconde au Louvre. Afin de parvenir à cette image-culte du Da Vinci Code, des centaines de milliers de visiteurs doivent passer le seuil de deux portes qui sont séparées par un énorme tableau lourdement encadré: les Noces de Cana de Véronèse. Euvre gigantesque et plutôt sombre, ce tableau fait face à la minuscule Mona Lisa, qui est à peine visible derrière son épais verre de protection «anti-fanatiques». À présent, notre visiteuse est véritablement stupéfaite. Dans cette machine hollywoodienne mettant en scène un mariage miraculeux, elle ne reconnaît en rien le fac-similé qu'elle a eu la chance de voir à la fin de l'année 2007 alors qu'elle avait été invitée par la Fondazione Cini sur l'île de San Giorgio à Venise. Elle se souvient vivement de l'œuvre en question, il s'agissait d'un tableau peint sur toile dont l'épaisseur et la profondeur étaient telles que l'on pouvait encore admirer les traits de pinceau de Véronèse et sentir les entailles aiguës opérées par les aides de camp de Napoléon afin d'arracher le tableau du mur, une bande à la fois, avant de l'enrouler comme un tapis et de l'envoyer à Paris en tant que butin de guerre, en 1797 - viol culturel dont tous les Vénitiens se souviennent encore aujourd'hui. Mais là, dans le réfectoire de Palladio, le tableau (oui, il s'agissait d'un tableau, même s'il avait été produit à l'aide de techniques numériques) possédait un sens très différent: il avait été installé à une hauteur différente, conforme à la vocation du lieu (une salle à manger). Il baignait subtilement dans la lumière naturelle provenant des grandes fenêtres situées à l'est et à l'ouest, de sorte que l'été, vers dix-sept heures, la luminosité dans la pièce coïncidait parfaitement avec la luminosité dans le tableau. Le tableau ne 


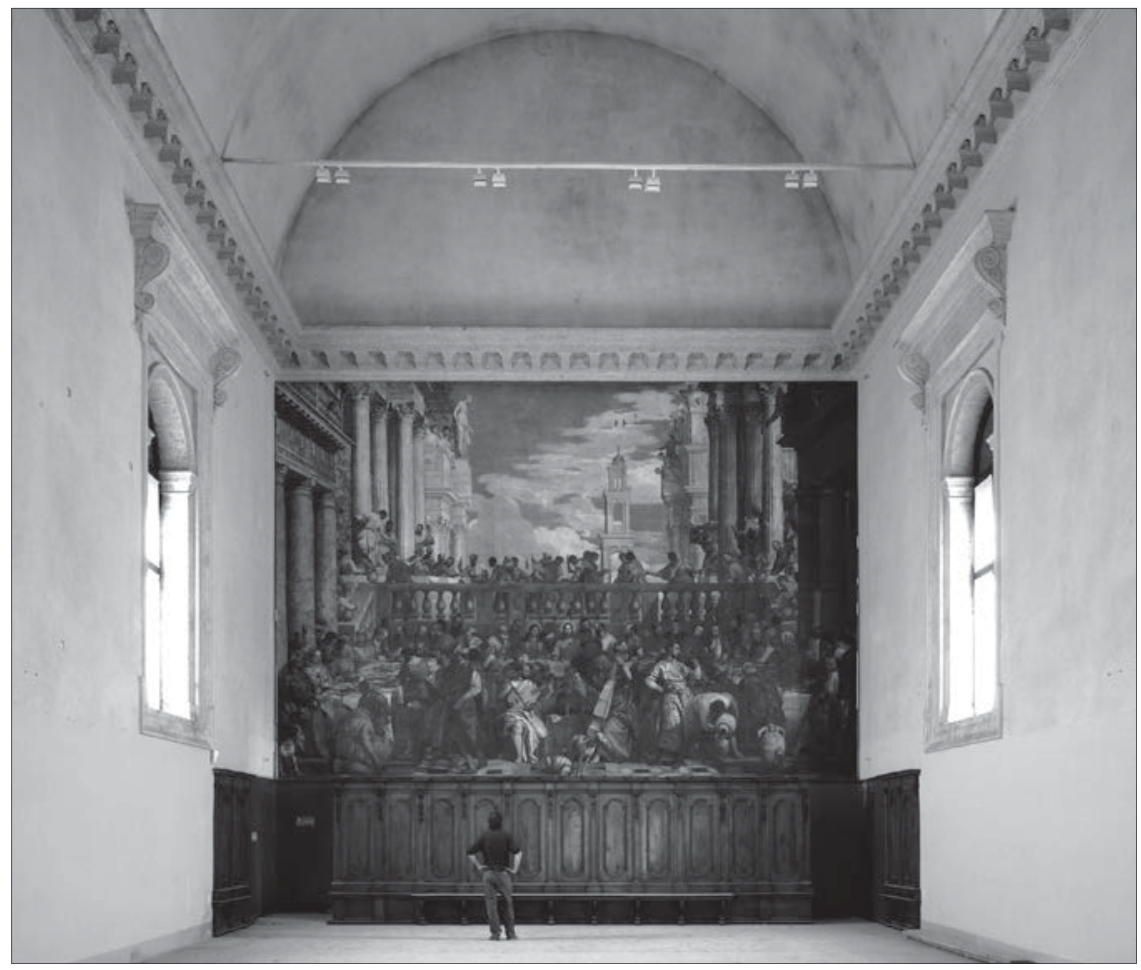

Fig. 1: Adam Lowe observant Les noces de Cana achevé dans le réfectoire palladien de San Giorgio. (C) Adam Lowe.

possédait pas de cadre. D’ailleurs - et cela est encore plus important -, il y avait une continuité admirable entre le tableau et le lieu, si bien que l'architecture de Palladio fusionnait avec l'espace peint de Véronèse, produisant ainsi dans le réfectoire des moines bénédictins une profondeur de champ en trompe-l'œil si réussie que l'on ne pouvait pas s'empêcher de s'avancer et de reculer et de circuler dans la pièce afin de pénétrer plus profondément dans le mystère de ce miracle (Fig. 1).

Or, ici, dans la salle de la Joconde, bien que toutes les parties du tableau soient ressemblantes (du moins dans le souvenir de notre visiteuse), le sens du tableau qu'elle avait tant admiré à Venise n'y est pas. Pourquoi le tableau est-il enchâssé dans cet encadrement doré si massif? Pourquoi voit-on des portes à chaque extrémité? Pourquoi est-il accroché si bas, rendant ridicule le balcon vénitien sur lequel les convives se réunissent? Les mariés, repoussés dans le coin gauche de l'image, semblent marginalisés, alors qu’à Venise ces figures étaient dotées d'une grande importance, explicitant une scène d'intrigue sexuelle qui 
évoquait un photogramme de film. Le sens de la composition est moins riche à Paris. Pourquoi cet éclairage zénithal si laid? À Venise, il n’y avait pas d'air climatisé; le tableau pouvait respirer par ses propres moyens, comme si Véronèse l'avait accroché pour qu'il puisse sécher. Enfin, ici les visiteurs ne peuvent pas circuler autour du tableau et réfléchir sur ces questions sans se heurter à ceux qui s'agglutinent momentanément autour de la Joconde (ils font la queue), tournant ainsi le dos au Véronèse.

Il s'agit là d'une dissonance cognitive terrible. Et pourtant, il ne fait aucun doute que ce tableau-ci, à Paris, est l'original. Aucune substitution n’a eu lieu, aucune supercherie - en dépit des nombreuses restaurations. Véronèse serait sans doute surpris de voir le tableau dans cet état, mais cela n'est pas la même chose que de la supercherie. Notre visiteuse se souvient parfaitement qu'à Venise, il était clairement indiqué: «Un fac-similé ». Et à San Giorgio, il y avait même une petite exposition visant à expliquer en détail le processus numérique fort complexe que l'atelier de Madrid, Factum Arte, avait employé afin de dématérialiser et re-matérialiser le gigantesque tableau parisien: en le numérisant d'abord au moyen de lasers, à raison d'une surface $\mathrm{A}_{4}$ à la fois, ensuite en le photographiant en autant de sections et en le numérisant à l'aide d'un scanner ${ }_{3} \mathrm{D}$ afin d'enregistrer sa toponymie, enfin, en fusionnant tous ces fichiers numériques, avant d'employer une imprimante fabriquée sur mesure pour appliquer des pigments sur un canevas apprêté avec du plâtre quasiment identique à celui employé par Véronèse. Serait-il possible que la version de Venise, ce fac-similé exposé en tant que tel, soit plus originale que l'original de Paris, se demande notre visiteuse? À présent, elle se souvient de cette conversation téléphonique avec une amie historienne de l'art française, qui lui avait reproché d'avoir passé autant de temps à San Giorgio devant la copie des Noces: «Tu perds ton temps devant ce faux Véronèse alors qu'à Venise il y a tellement d'originaux du même peintre! », lui avait dit son amie. À cela elle avait répondu, sans trop savoir ce qu'elle disait: «Mais viens donc le voir et le constater par toi-même, aucune description ne peut remplacer l'expérience de voir cet original... oh là, je voulais dire, n'est-ce pas cela, précisément, la définition même de "l'aura"?... Indéniablement, pour elle, l'aura de l'original avait migré du Louvre vers San Giorgio : la preuve tenant au fait qu'il fallait venir voir l'original pour le constater. Quel contraste dramatique, se ditelle, entre le Véronèse et les Ambassadeurs, ce dernier prétendant être l'original afin de cacher le fait qu'il s'agit d'une copie très coûteuse d'une de ses copies de mauvaise qualité!

«Mais il ne s'agit pas de l'original, ce n'est qu'un fac-similé! » Combien de fois avons-nous entendu cette réplique devant la reproduction parfaite d'un 
tableau? La chose ne fait aucun doute: notre époque est obsédée par l'original. Seul l'original possède une aura, qualité mystérieuse et mystique faisant défaut à toute version de seconde main. Or, paradoxalement, cette obsession de l'original augmente proportionnellement à la disponibilité et à l'accessibilité d'un nombre croissant de copies dont la qualité s’améliore sans cesse. Si nous consacrons autant d'effort à la recherche de l'original - pour des raisons liées à l'archéologie et au marketing -, c'est bien parce que la possibilité de créer des copies n’a jamais été si manifeste. S'il n'existait aucune copie de la Joconde, déploierions-nous autant d'énergie à venir voir l'original - et serions-nous si enclins à émettre toutes ces théories de conspiration afin de déterminer si la version exposée sous verre et protégée par un système d'alarme sophistiqué constitue la surface originale peinte de la main de Léonard? Autrement dit, l'intensité de la quête de l'original dépend de l'intensité de la passion et de l'intérêt déclenchés par les copies. Pas de copie, pas d'original. Pour marquer une œuvre du sceau de l'originalité, vous devez appliquer sur sa surface une pression énorme que seule un très grand nombre de reproductions permet d'exercer.

En dépit de la réaction spontanée - «Mais ce n’est qu’un fac-similé»-, nous ne devrions pas trancher la question trop rapidement quant à la valeur de l'original ou de sa reproduction. Dès lors, le véritable phénomène qu'il convient d'expliquer n'est pas celui du tracé point par point d'une version ayant été séparée du reste de ses copies. En effet, il importe plutôt de saisir l'ensemble lui-même, celui qui est constitué d'un ou de plusieurs originaux ainsi que d'une biographie qui ne cesse d'être réécrite. Il ne s'agit pas d'un cas de «et/ou» mais de «et, et». La quête centenaire de la source du Nil n'a-t-elle pas été si passionnante parce que le Nil aboutit à un delta si vaste? Pour filer la métaphore: dans cette réflexion nous voulons nous conduire comme des hydrographes cherchant à déployer tout le bassin hydrographique d'une rivière, plutôt que de nous borner à approfondir la source originale. Nous ne devrions pas comparer une œuvre d'art à un locus isolé, mais plutôt à l'ensemble d'un bassin hydrographique, avec ses estuaires, ses nombreux affluents, ses rapides turbulents, ses nombreux méandres et, bien sûr, ses multiples sources cachées.

Afin de nommer ce bassin hydrographique, nous emploierons le terme de trajectoire. Une œuvre d'art - peu importe les matériaux ayant servi à la fabriquer - possède une trajectoire ou, pour employer une autre expression que les anthropologues ont popularisée: une carrière ${ }^{1}$. Dans ce texte, nous souhaitons

1. Voir Arjun Appadurai (dir.), The Social Life of Things. Commodities in Cultural Perspective, Cambridge, Cambridge University Press, 1986. Voir également Miguel 
approfondir la trajectoire ou la carrière de l'œuvre d'art, de sorte que nous passons d'une question équivoque: «Est-ce un original ou seulement une copie? » à une interrogation que nous tenons pour décisive, surtout dans le contexte actuel de la reproduction numérique: "L'œuvre a-t-elle été bien ou mal reproduite?» La raison pour laquelle cette seconde question nous semble si importante tient au fait que la qualité, la conservation, la continuité, la subsistance et l'appropriation de l'original dépendent entièrement de la distinction entre une bonne et une mauvaise reproduction. Nous postulons qu'un original mal reproduit risque de disparaître, alors qu'une copie tenant bien compte de l'original pourrait continuer à mettre en valeur son originalité et générer de nouvelles copies. Voilà la raison pour laquelle nous cherchons à démontrer que les fac-similés, surtout ceux qui sont issus de techniques (numériques) complexes, s'avèrent être la façon la plus féconde d'explorer l'original et même de contribuer à redéfinir ce qu'est l'originalité.

Afin que le lecteur mette hors jeu la volonté d'identification de l'original et qu'il prête attention à la question de la qualité de la reproduction, il convient de rappeler que le mot «copie » n’est pas forcément péjoratif, puisqu'il possède la même étymologie que « copieux »; dès lors, il désigne une source d'abondance. Il n’y a rien d'inférieur dans la notion de copie, seulement une preuve de fécondité. Loriginalité est-elle suffisamment féconde pour produire une abondance de copies? Dans cette optique, nous renvoyons à l'ancienne image de la corne d'abondance afin de donner une première concrétisation de la notion abstraite de trajectoire. La corne d'abondance: une corne de bélier torsadée possédant une extrémité pointue - l'original - et une bouche béante dégorgeant à volonté un flot de richesses (tout cela grâce à Zeus). En fait, cette idée d'une pluralité de copies toutes liées à un original ne devrait pas surprendre; dire qu'une œuvre d'art est originale ne signifie rien d'autre à part qu'elle est l'origine d'une longue lignée. Une entité sans progéniture, sans reproduction et sans héritier n’est pas dite originale mais stérile ou improductive. Ainsi vaudrait-il mieux remplacer la question: "Cette ouvre isolée est-elle un original ou un fac-similé ?» par celle-ci: "Ce segment dans la trajectoire de l'œeuvre est-il stérile ou fécond?»

Affirmer qu'une œuvre d'art devient de plus en plus originale grâce à la qualité et à l'abondance de ses copies n'est pas du tout curieux : cela est vrai de la trajectoire de n'importe quel ensemble d'interprétations. Si les chants de l'Iliade étaient restés enlisés dans un petit village d’Asie Mineure, Homère ne serait pas

Tamen, Friends of Interpretable Objects, Cambridge (Mass.), Harvard University Press, 2001. 
envisagé aujourd'hui comme un auteur (collectif) d'une si grande originalité. C'est bien en raison - et non pas en dépit - des milliers et milliers de répétitions et variations qu'ont connues ces chants que nous sommes émus, en feuilletant n'importe quelle copie de l'Iliade, de la fécondité sans borne de l'original. Nous imputons à l'auteur (même si son existence ne peut pas être déterminée) le pouvoir que possède chacune des réinterprétations successives en disant que celles-ci «étaient » toutes « déjà » dans le Ur-text, ne serait-ce qu’en «puissance ». Or nous savons que cela est à la fois faux (il est impossible que mon interprétation corresponde à celle des Grecs anciens) et tout à fait vrai, dans la mesure où j'ajoute volontairement ma petite extension, étendant ainsi la fécondité «illimitée » de ce phénomène collectif que nous nommons «Iliade». Si ce texte possède ce caractère si illimité, c'est bien parce que je puis repousser la limite encore un peu plus loin. Cela ne signifie pas qu'il n'y a rien d'«intrinsèquement grand» dans les premières versions de ce grand poème et que pour pénétrer dans cette grandeur intrinsèque, nous devons tenir compte de toutes les versions successives, des adaptations et des ajustements. Il n'est rien de plus ordinaire que ce mécanisme: Abraham devient le père de son peuple, qui est «aussi nombreux que les grains de sable», en raison de sa descendance. Avant la naissance d'Isaac, Abraham était un homme méprisé et stérile. Qu'il soit devenu le «Père de trois religions » est dû aux péripéties d'Isaac et, par la suite, à ce qui est advenu à chacun de ses fils et filles. Voilà « l'incroyable responsabilité du lecteur », comme l'a dit Charles Péguy de façon éloquente, puisque ce processus est parfaitement réversible; si nous cessons d'interpréter, si nous cessons de répéter, si nous cessons de reproduire, l'existence même de l'original est en jeu. Il se pourrait que celui-ci cesse de produire des copies abondantes et qu'il disparaisse tranquillement ${ }^{2}$.

Il n'est pourtant pas difficile de remettre en cause la qualité de l'ensemble de la trajectoire lorsqu'il est question de pratiques artistiques à caractère performatif comme la danse, la musique ou le théâtre. Pourquoi est-il si difficile d'en faire autant vis-à-vis de la reproduction d'un tableau ou vis-à-vis d'un meuble, d'un édifice ou d'une sculpture? Cela constitue la première question que nous souhaitons éclaircir.

En assistant à une représentation du Roi Lear, il ne viendrait à l'esprit de personne de dire: «Mais ce n'est pas l'original, ce n'est qu'une représentation!» En effet. Voilà le sens du geste consistant à jouer le Roi Lear: il s'agit de le rejouer. Dans le cas d'une œuvre à caractère performatif, tout le monde est prêt

2. Voir les commentaires sur Péguy dans Gilles Deleuze, Différence et répétition, Paris, Les Presses Universitaires de France, coll. «Epiméthée », 1968. 
à tenir compte de l'ensemble de la trajectoire, des premières représentations jusqu'aux plus récentes, en passant par les innombrables «revivals». Il n'y a rien d'extraordinaire dans le constat qu'«une bonne représentation du Roi Lear» est un moment, un segment, dans la carrière de l'œuvre intitulée Le Roi Lear, dont l'idéal platonicien absolu n’a jamais été observé et ne sera jamais circonscrit par personne. D'ailleurs, ce n'est pas faire preuve de grande sophistication que d'admettre que la quête de «la» première représentation, l'originale, celle de Shakespeare «lui même», sera décevante, dans la mesure où il y a eu plusieurs premières ainsi que des dizaines de versions écrites de la pièce, avec leurs gloses et variations sans fin. Nous semblons parfaitement heureux de nous passionner pour la découverte antidramatique de la source d'une rivière importante, même s'il s'agit d'une source modeste et à peine visible sous des herbes couvertes de mousse. Enfin, et cela est encore plus important, les spectateurs n'hésitent pas du tout à juger une nouvelle version qu' ils ont sous les yeux à la lumière du principe : «Est-ce bien ou mal (re)joué? » Bien qu'il leur arrive d'émettre des jugements très variés, certains étant scandalisés par des innovations qui leur semblent révoltantes ("Pourquoi le roi Lear disparaît-il dans un sous-marin?») ou ennuyés par la trop fréquente répétition de clichés, ils n’ont aucune difficulté à envisager que ce moment dans la totalité de la carrière de tous les Roi Lear (au pluriel) successifs devrait être jugé sur son propre mérite et non pas en effectuant une comparaison mimétique avec la première représentation (au demeurant tout à fait inaccessible) par la troupe de Shakespeare en telle ou telle année. C'est ce qui est là sur scène, maintenant, sous nos yeux, qui motive notre jugement, et très certainement pas le degré de ressemblance avec un autre Ur-événement qui est en retrait (même si ce que nous tenons pour le vrai «Roi Lear» demeure en arrière-plan à chaque fois que nous émettons un jugement à propos d'une de ses représentations). Ainsi, il saute aux yeux, dans le cas des arts à caractère performatif au moins, que chaque nouvelle version court le risque de perdre l'original - ou de le récupérer.

Dès lors, nous nous affranchissons de toute comparaison avec un «original» et il est parfaitement acceptable d'évaluer une nouvelle réalisation en disant: "Ie n'aurais jamais pu anticiper cela; cela est entièrement différent des représentations antérieures; cela se distingue de la façon dont Shakespeare jouait la pièce, et pourtant je comprends à présent ce dont il a toujours été question dans cette pièce!» Tout se passe comme si quelques-uns des revivals - les bons - avaient su extraire de l'original des traits nouveaux qui se trouvaient potentiellement dans la source, mais qui sont demeurés invisibles jusqu'au moment présent où ils animent l'esprit des spectateurs. Ainsi, bien que la pièce ne soit 
pas évaluée en fonction de sa ressemblance mimétique avec un modèle idéal, il est clair, et nous croyons que tout le monde sera de notre avis, qu'en raison des gestes faits par un successeur tardif, le génie de Shakespeare aura atteint un nouveau degré d'originalité grâce à l'exploit extraordinaire de cette reproduction fidèle (et non pas mimétique). Lorigine est là à nouveau, même si elle diffère de ce qu'elle a été. Et le même phénomène pourrait avoir lieu avec n’importe quelle œuvre musicale ou chorégraphique. L'exclamation: «Cela est tellement original! » attribuée à une nouvelle représentation ne recouvre pas une section de la trajectoire (et surtout pas la première Ur-version) mais le degré de fécondité de l'ensemble de la corne d'abondance. Dans l'art à caractère performatif, l'aura ne cesse de migrer et il lui arrive de resurgir soudainement... ou de disparaître au complet. Lorsqu'un grand nombre de mauvaises répétitions affaiblissent le degré de fécondité d'une œuvre au point de délaisser l'original lui-même, celuici cesse de fonctionner à titre de point d'amorce d'une succession. Une telle $œ u v r e$ d'art s'éteint comme une lignée sans descendant. Comme une rivière privée successivement de tous ses affluents, au point de rétrécir et de ne devenir qu'un minuscule filet, l'œuvre d'art est réduite à sa taille « originale », c'est-à-dire à quelque chose de très petit. Dans cet état, elle n'a pas encore été copieusement copiée, c'est-à-dire constamment réinterprétée et relancée. L'œuvre a ainsi perdu son aura pour de bon.

Pourquoi est-il si difficile d'émettre le même type de jugement devant un tableau, une sculpture ou un édifice? Pourquoi ne pas dire, par exemple, que le fac-similé des Noces de Cana de Véronèse a été rejoué, répété, ou réanimé grâce à une nouvelle interprétation, celle qui a eu lieu à Venise en 2007 sous l'égide de Factum Arte, tout comme Les Troyens de Hector Berlioz ont enfin été joués à Covent Garden à Londres pour la première fois, en 1969, sous la direction de Colin Davis (un exploit que le pauvre Berlioz n’a jamais été en mesure de réaliser, puisqu'il lui manquait l'argent et l'orchestre pour jouer son œuvre originale au complet...)? Et pourtant, ce qui semble aller de soi pour l'art à caractère performatif semble encore tiré par les cheveux dans le champ des arts visuels. Si nous affirmons que l'œuvre Les Noces de Cana a été « déployée à nouveau » à San Giorgio, d'aucuns diront sans hésiter : «Mais l'original est à Paris! Le tableau de San Giorgio n'est qu'un fac-similé!» Une impression de falsification, de supercherie, voire de trahison s'est introduite dans le débat; et celle-ci serait somme toute assez absurde s'il s'agissait non pas d'un tableau mais d'une œuvre à caractère performatif (bien qu'il soit tout à fait possible d'affirmer d'une mauvaise troupe qu'elle a travesti Shakespeare). Il semble presque impossible d'avancer que le fac-similé des Noces de Cana de Véronèse n'est pas de l'ordre de la falsification, 
mais constitue plutôt une étape dans le processus de confirmation de la contribution de l'artiste, une partie de la biographie de son œuvre en cours de rédaction.

De toute évidence, une des raisons motivant ces façons inégales d'envisager la reproduction tient à ce que nous pourrions appeler la différentielle de résistance de chaque segment constituant la trajectoire. Dans son essai, dont la célébrité est démesurée et où il ne se prive pas de faire intervenir l'histoire de l'art sous la forme d'un épais brouillard de mysticisme, Walter Benjamin a cerné cet écart technologique en l'appelant «reproduction mécanisée ${ }^{3}$ ». Dans le cas de l'art à caractère performatif, chaque version est tout aussi difficile à produire, et tout aussi coûteuse, que la précédente (ou plutôt, chaque version devient de plus en plus dispendieuse, et cette inflation des coûts est très certainement plus importante qu'au temps de Shakespeare - pensez au salaire des gardiens et à toutes les normes de santé et de sécurité !). Ce n'est pas parce qu'il y a eu un nombre incalculable de représentations du Roi Lear que celle que vous vous apprêtez à réaliser sera plus facile à financer. Le coût marginal sera exactement le même - à la différence près que le public saura en quoi consiste « un Roi Lear », puisqu'il arrivera avec un bagage sans fin de préjugés et de critères relatifs à la façon dont il faut jouer la pièce (et cela constitue une épée à double tranchant, comme le sait tout metteur en scène). Cela constitue la raison technique pour laquelle, dans le cas des arts à caractère performatif, nous ne distinguons pas entre un original et une copie, mais plutôt entre des versions successives de la même pièce, chacune étant désignée par l'appellation «version $n$ ", «version $n+1 »$, «version $n+2 »$, etc. Et c'est également la raison pour laquelle la vraie pièce «Le Roi Lear» ne se situe nulle part spécifiquement (en tout cas pas au commencement); plutôt, elle consiste en un nom attribué à l'ensemble de la corne d'abondance elle-même (même s'il arrive à chaque spectateur ou spectatrice de s'attacher à une expérience unique, appartenant à l'histoire personnelle de chacun, qui a lieu lorsqu'une nouvelle réalisation particulièrement «vivante » fait en sorte que le génie du vrai Roi Lear se «concrétise » avec plus de force que dans les représentations antérieures ou postérieures). Dans ces cas, la trajectoire est constituée de segments composés, pour ainsi dire, de la même substance ou, du moins, mobilisant des ressources semblables.

Il en va autrement lorsqu'on envisage, par exemple, un tableau. Puisqu'un tableau reste dans le même encadrement alors qu'il continue à être encodé par

3. Walter Benjamin, «L'œuvre d'art à l'époque de sa reproductibilité technique» [dernière version de 1939], dans (Euvres III, trad. Maurice de Gandillac, Paris, Gallimard, coll. «Folio essais », 2000, p. 269-316. 
les mêmes pigments et demeure dans la même institution, nous ne pouvons pas nous empêcher de croire que chaque reproduction sera d'autant plus facile à réaliser et qu'il n'y aura donc pas lieu de comparer les divers segments de la trajectoire entre eux quant à la question de leur qualité. Voilà la raison pour laquelle l'aura semble définitivement liée à une seule version : la version autographe. Cela est sans doute vrai d'un point de vue superficiel: si vous prenez une photo des Noces de Cana à Paris à l'aide de votre appareil numérique, il ne viendrait à l'esprit de personne de mettre sur un pied d'égalité cette pâle copie apparaissant sur l'écran de votre ordinateur et le tableau de $67 \mathrm{~m}^{2}$ accroché au Louvre... Si vous affirmiez que votre image était «tout aussi belle que l'original», tout le monde hausserait les épaules avec pitié, et avec raison.

Et pourtant, la distance séparant la «version $n$ », que nous désignons comme étant «l'original», et la «version $n+l$ », que nous appelons «la simple copie», dépend aussi bien de la différentielle des efforts, des coûts et des techniques que de toute différence substantielle existant entre les versions successives d'un même tableau. Autrement dit, ce n'est pas en raison d'une quelconque qualité intrinsèque de la peinture que nous sommes enclins à poser un écart si grand entre les originaux et les copies - ce n'est pas parce que la peinture est plus «matérielle» (un opéra ou une pièce de théâtre sont tout aussi «matériels» que les pigments sur une toile) -, cela découle plutôt des différences entre les techniques employées dans chaque segment de la trajectoire. Alors que dans les arts à caractère performatif, ces dernières sont grosso modo homogènes (chaque nouvelle réalisation faisant appel au même éventail de techniques), la carrière d'un tableau ou d'une sculpture dépend de segments fort hétérogènes qui varient considérablement sur le plan de l'intensité des efforts déployés en cours de route. Nous souhaitons démontrer que c'est très précisément cette asymétrie qui empêche souvent celui qui regarde d'affirmer que le tableau des Noces de Cana de Paris a été «réimprimé» ou «déployé à nouveau » à Venise. Et c'est très certainement cette même présupposition qui enrageât à ce point l'historienne de l'art française qui reprochait à son amie de passer autant de temps à San Giorgio au lieu de se consacrer aux «vrais Véronèse ». La distinction que nous faisons couramment entre l'original et la simple copie occulte un processus d'un autre ordre qui a trait à l'équipement technique, au soin et à l'intensité de la recherche de l'originalité conduisant d'une version à une autre. Avant qu'un fac-similé puisse se faire valoir en tant que bonne ou mauvaise reconstitution de l'original, nous le dévalorisons, puisque nous l'associons à un écart dans les techniques de reproduction, écart résultant d'une mauvaise conception de la photographie comme index de la réalité. 
Nous donnons pour preuve de cette affirmation ce qui advient à notre quête de l'originalité lorsque nous modifions ce différentiel - et cela est de plus en plus facile dans la nouvelle ère numérique. Que cela ne soit pas limité aux arts à caractère performatif est évident, surtout si on se réfère à la pratique de la copie de manuscrits. Avant l'invention de l'imprimerie, le coût marginal lié à la production d'une copie supplémentaire était tout à fait identique au coût de production de l'avant-dernière copie - situation à laquelle nous retournons à présent avec les copies numériques. Dans le scriptorium d'un monastère, tout exemplaire constituait en soi une copie; il ne serait venu à l'esprit d'aucun copiste d'affirmer que cet exemplaire est l'original alors que cet autre est seulement une copie - c'étaient tous des fac-similés -, bien que les copistes mettaient le plus grand soin à distinguer une copie inférieure d'une meilleure version, plus ancienne et mieux enluminée. Encore une fois, dans ce contexte, l'aura pouvait se déplacer et migrer vers la plus récente copie, celle qui avait été effectuée avec brio sur un des meilleurs parchemins et avait fait l'objet d'une lecture comparative en fonction des meilleures sources anciennes. Naturellement, après l'invention de l'imprimerie, le coût marginal d'une copie supplémentaire est devenu négligeable par comparaison avec les techniques et le temps requis pour la fabrication d'un manuscrit; c'est ainsi, et seulement ainsi, qu'une énorme distance s'est, à juste titre, introduite entre une partie de la trajectoire - le manuscrit autographe, qui deviendra désormais l'ORIGINAL - et le tirage - lequel, à partir de ce moment, sera envisagé comme n'englobant que des copies (jusqu'au moment, certes, où l'art de la bibliophilie nous aura révélé les subtilités sans fin distinguant chaque tirage successif, et où le champ de l'expertise médicolégale numérique nous aura permis de dater et de classer ces copies).

Pour prouver que l'aura - en ce qui concerne sa capacité d'être extraite du flux des copies (ou de rester enlisée dans un segment de la trajectoire) - dépend de façon cruciale de l'hétérogénéité des techniques employées dans les segments successifs, rien de mieux que d'envisager ce qui advient au livre ORIGINAL maintenant que nous sommes tous assis dans ce lieu du «couper-coller », ce scriptorium mondial que nous appelons le Web. Puisqu'il n'y a plus de différence importante permettant de distinguer les techniques employées dans chaque réactualisation d'un segment d'hypertexte original, nous acceptons spontanément l'idée qu'il n'y a aucune différence considérable entre une version, que nous qualifiions auparavant d'«originale», et les versions ultérieures, celles taxées de n’être «que des copies». Nous désignons sans hésiter les instances successives d'un «même» argument à l'aide des expressions «version 1 », «version 2 », « version $n$ », alors que la notion d'auteur est devenue tout aussi floue que celle d'aura 
- pour ne pas mentionner le sort réservé aux redevances des droits d'auteur. D’où la popularité des scriptoria collectifs comme Wikipédia. En effet, Benjamin a confondu la notion de «reproduction mécanisée» avec celle de l'inégalité des techniques employées au sein d'une trajectoire. Il importe peu qu'une reproduction soit issue d'un processus plus ou moins mécanisé. Dès lors qu'il n'y a plus d'écart important permettant de différencier le processus de production de la version $n$ de celui de la version $n+n$, distinguer clairement entre un original et ses reproductions devient une tâche moins importante - alors l'aura se met à hésiter, elle n’arrive pas à se décider quant au lieu où elle devrait se poser.

Quoi qu'il en soit, est-il possible d'envisager la migration de l'aura dans le cas de la reproduction ou de la réinterprétation disons, d'un tableau? Après tout, nous avons amorcé notre recherche en comparant les Noces de Cana et les Ambassadeurs; et notre réflexion aurait été d'un tout autre ordre si nous nous étions arrêtés aux arts à caractère performatif. Nous ne pouvons pas nous empêcher de soupçonner qu'il y a, en peinture, en architecture, en sculpture, et dans le champ des objets en général, une espèce d'obstination tenace consistant à associer de façon inséparable un lieu, un original et une aura.

Cependant, il convient d'abord de constater que la différence entre les arts à caractère performatif et les autres champs artistiques n'est pas aussi radicale qu'il semble: un tableau doit toujours être reproduit, c'est-à-dire qu'il est toujours une re-production de lui-même, même s'il semble demeurer le même et rester au même endroit. Ou plutôt, aucun tableau ne demeure le même au même endroit sans subir quelque instance de reproduction. L'existence précède l'essence, même pour les tableaux. Afin de posséder une substance durable, les tableaux doivent être à même de subsister. Ce réquisit est bien connu des conservateurs à travers le monde: un tableau doit être remis dans un nouvel encadrement, il doit être épousseté, parfois il doit être restauré, éclairé, et il doit être représenté dans des salles différentes et accompagné de différents tableaux, sur des murs différents, et il doit pouvoir être inséré dans différents récits, dans des catalogues différents, tout en subissant des variations quant à sa valeur assurée et quant à son prix. Ainsi, même si un tableau ne fait jamais l'objet d'un prêt et continue à survivre au sein d'un même cadre institutionnel sans subir d'importantes restaurations, il possède tout de même une carrière ; afin de subsister et de redevenir visible, il faut qu'on s'occupe de lui. Si personne ne se soucie d'un tableau donné, ce dernier se retrouvera bientôt dans une cave, accumulant de la poussière, ou sera vendu à rabais ou découpé en morceaux et perdu à jamais. Voilà ce qui motive toutes ces restaurations: si l'on ne fait rien, le temps dévorera les tableaux et l'immeuble dans lequel ces derniers sont conservés tombera très certainement en ruine, tout 
comme les institutions ayant la charge des tableaux se décomposeront. Au cas où vous douteriez encore de cela, imaginez vos œuvres précieuses conservées au Musée national de Kaboul... Pour qu'une œuvre d'art puisse survivre, il lui faut une écologie tout aussi complexe que celle permettant de préserver la dimension naturelle d'un parc naturel ${ }^{4}$.

Si la nécessité de la reproduction était acceptée, nous serions peut-être à même de convaincre le lecteur que la question importante n'est pas celle de la différence entre original et fac-similés, mais plutôt celle ayant trait à la distinction entre une bonne et une mauvaise reproduction. Les Ambassadeurs n'a pas été irrévocablement effacé en raison de la négligence des conservateurs; au contraire, la disparition de ce tableau s'explique par un zèle excessif visant à le «reproduire». En effet, dans le cas des Ambassadeurs, les conservateurs ont confondu un des attributs généraux de toute œuvre d'art - afin de survivre une œuvre doit être reproduite d'une façon ou d'une autre -, avec une conception très restreinte de la reproduction qui est celle des affiches photographiques. Ainsi ont-ils mis hors jeu plusieurs autres façons de reproduire un tableau. À titre d'exemple, ils auraient pu produire un fac-similé parfait en captant toute la surface de l'œuvre en trois dimensions; ainsi auraient-ils restauré la copie au lieu de travailler sur l'œuvre elle-même. L'auraient-ils fait, ils auraient pu demander à plusieurs historiens de l'art aux points de vue divergents de suggérer différentes méthodes pour restaurer la copie, en vue de constituer une exposition à partir de ces résultats. Leur crime n'est pas d'avoir proposé une reproduction du Holbein, au lieu du Holbein lui-même, aux visiteurs de la National Gallery. De même que Les Ambassadeurs subsiste quelque part derrière toutes les restaurations successives, de même Le Roi Lear perdure dans chaque réalisation de cette pièce, alors que l'œuvre confère ou refuse sa dimension auratique en fonction du mérite de chaque réalisation. Le crime des conservateurs est plutôt d'avoir restreint le champ des techniques de reproduction et d'en avoir retenu la technique la plus infertile: la photographie. À leurs yeux, c’est comme si la peinture n’était pas un matériau tactile mais une image éthérée, un schème que l’on peut extraire de sa matérialité et télécharger dans n’importe quelle reproduction sans pour autant perdre sa substance. En effet, un documentaire tristement révélateur nous montre les coupables en train de restaurer le Holbein; leur modèle s'avère être des photographies de l'original. Ainsi, sur la base de critères subjectifs, ils décident ce qui appartient à l'original, ce qui s'est désagrégé, et ce qui y a été ajouté. Bref,

4. Voir David Western, In the Dust of Kilimanjaro, New York, Shearwater Books / Island Press, 1997. 
ils conçoivent le tableau comme une série de couches hétérogènes pouvant être superposées ou retirées au besoin - processus évoquant davantage l'univers de la chirurgie plastique que celui de l'enquête médicolégale.

Ainsi, ce qui paraît si extraordinaire lorsque nous comparons le sort des Ambassadeurs avec celui des Noces de Cana n'est pas tant que ces deux tableaux ont recours à la reproduction - dans les deux cas la reproduction est une nécessité de l'existence même des œuvres -, mais que le premier s'appuie sur une conception de la reproduction dans laquelle l'original disparaît à jamais, alors que le second ajoute de l'originalité à la version originale tout en proposant de nouvelles dimensions et ce, sans mettre l'avant-dernière version en péril - sans même la toucher, grâce aux processus délicats employés afin de l'enregistrer.

On se demandera peut-être : comment peut-on ajouter de l'originalité à une œuvre? Une réponse évidente serait: en transportant la nouvelle version dans le lieu d'origine. La dissonance cognitive caractérisant l'expérience du spectateur dans la salle de la Joconde tient, du moins en partie, au fait que dans le réfectoire de Palladio, chaque petit détail des Noces de Cana est doté d'un sens qui est entièrement perdu au Louvre, étant donné l’accrochage maladroit de la version $n-1$ à Paris. Autrement dit, l'originalité n'est pas quelque chose advenant à une œuvre d'art en bloc; au contraire, l'originalité est constituée de différentes composantes, chacune entrant en relation avec les autres afin de composer une totalité complexe. Des nouveaux procédés de reproduction nous permettent d'appréhender ces éléments ainsi que leurs relations sous un nouveau jour. Placer une œuvre dans le lieu pour lequel elle a été conçue dans tous ses détails constitue très certainement un aspect - un élément - de ce que nous entendons par l'original. Dans cette perspective, il n'y a aucun doute que le fac-similé des Noces de Cana tient maintenant lieu de l'original, tandis que la version du Louvre a été pour le moins privée de cet avantage comparatif.

Il faut prendre garde à ne pas entendre la notion du «lieu original » de façon trop mystique dans le cas du Véronèse, car le réfectoire dans lequel le fac-similé a été installé est lui-même une reconstruction. En vous référant aux photographies prises dans les années 1950, vous constaterez qu'à cette époque le plancher original avait disparu et un autre plancher avait été construit au niveau des fenêtres. Le niveau supérieur abritait un théâtre et le niveau inférieur, un atelier de menuiserie - bref, l'ensemble de l'espace avait subi des transformations. Il avait été reconstruit dans les années 1950, mais le plâtre et le plancher ne concordaient plus avec le plan d'origine et le lieu était dépourvu des boiseries qui avaient pour fonction d'encadrer l'espace en y ajoutant les touches finales et en en soulignant les proportions. Dans cet état dénudé, le réfectoire ressemblait 
à un espace protestant typique qui semblait se moquer de l'absence du tableau de Véronèse, chef-d'œuvre de la Contre-Réforme. Aujourd'hui, l'effet que produit le fac-similé est tel que des rumeurs circulent selon lesquelles le retour du tableau aurait déclenché un plan de restauration visant à réaménager l'espace rétrospectivement, afin qu'il retrouve sa gloire passée. Un fac-similé d'un original ayant subi d'importantes restaurations, situé maintenant dans un nouveau lieu, aurait fait en sorte que des nouveaux éléments fussent ajoutés à un original dans son lieu d'origine, qui est en partie un fac-similé de lui-même. Autrefois l'originalité semblait si simple...

Nous pourrions avancer la même chose au sujet de la disponibilité. Au Louvre, les visiteurs sont manifestement frustrés de ne pas pouvoir balayer les Noces de Cana du regard sans se heurter aux fanas de la Joconde. Le tableau de Véronèse contient en soi tellement de détails et d'éléments imprévus que l'on ne peut pas le contempler sans s'accorder le temps d'approfondir son sens, ses implications, et les raisons motivant l'importance que l'œuvre continue de revêtir. Que signifie le geste de consacrer une ouvre s'il est impossible de contempler sa dimension auratique? Voici un autre élément que l'on peut retenir et distinguer de tous les autres. En fait, cette composante de l'originalité n'est pas forcément liée à l'originalité du lieu : il se pourrait que la meilleure preuve de cela soit le fac-similé de la chambre mortuaire de la tombe de Thoutmôsis III dans la Vallée des Rois 5 . Ce monument contient le premier texte intégral de l'Amdouat à figurer dans une tombe pharaonique. L'Amdouat est un récit complexe intégrant art, poésie, science et religion et se veut un compte rendu cohérent de la vie après la mort. La tombe n’a pas été conçue pour être visitée et les conditions matérielles et climatiques à l'intérieur du monument ne sont pas compatibles avec le tourisme de masse. Par conséquent, la tombe est en train de se détériorer rapidement et des panneaux en verre y ont été installés afin de protéger les murs de dommages accidentels et de l'usure. Cependant, ces interventions ont changé la vocation première du monument et empêchent les visiteurs d'étudier le texte de façon détaillée et d'apprécier l'ambiance spécifique du lieu. Les expositions qui présentent le fac-similé et mettent l'Amduat en contexte ont été visitées par des millions de personnes en Amérique du Nord et en Europe. Le fac-similé délocalisé a

5. Le fac-similé de la tombe (dans son état de conservation actuel, mais sans les éléments qui ont transformé le monument en musée) a fait l'objet de publications détaillées par l'égyptologue Erik Hornung et le psychologue Theodor Abt, sous forme de livres et de films. Voir Erik Hornung et Theodor Abt, The Dark Hours of the Sun: The Amduat in the Tomb of Thutmose III, DVD, publié par Factum Arte, 2005; et Erik Hornung et al., Immortal Pharaoh: The Tomb of Thutmose III, Madrid, Factum Arte, 2006. 
motivé les raisons mêmes de sa persistante importance, il a transformé les visiteurs en agents proactifs dans la campagne de conservation de la tombe, et il pourrait faire partie d'une politique à long terme visant à sauvegarder la version n-1 et à ne la rendre accessible qu'aux spécialistes dans le cadre de projets d'étude continus et à des fins de contrôle. Vous voyez? Chacune des composantes constituant ce que nous entendons par un véritable original se met à se déplacer à des vitesses différentes au sein de la trajectoire et commence à cartographier ce que nous avons appelé le bassin hydrographique de l'œuvre d'art.

Le troisième élément de l'originalité a trait aux propriétés de la surface de l'œuvre. Bien souvent, les restaurateurs travestissent la matérialité de l'original qu'ils se donnent pour tâche de protéger alors qu'ils réduisent la matière à la forme, puisqu'ils confondent tridimensionnalité et bidimensionnalité. S'il est une dimension de la reproduction que les techniques numériques ont complètement modifiée, c'est très certainement la capacité de capter les aspects tridimensionnels les plus infimes d'une œuvre, sans pour autant mettre l'œuvre en péril. Trop peu de chercheurs se souviennent que, dans ses débuts, le British Museum produisait des moulages en plâtre de ses objets et que le premier catalogue de l'institution contenait une liste des copies que le musée mettait en vente. Cela a trop souvent été oublié car la collection de moulages a été liquidée à la fin du $20^{\mathrm{e}}$ siècle; dès lors, des informations très précieuses portant sur la surface des œuvres au moment où celles-ci intégraient la collection ont été perdues. Plusieurs de ces plâtres contenaient encore de la peinture qui s'était détachée pendant le processus de moulage. D’ailleurs, les restaurations subséquentes des originaux ont modifié de façon dramatique la surface et l'apparence de plusieurs de ces objets. Ainsi, la question de la matérialité de l'œuvre d'art dépend de trajectoires fort complexes. En apprenant la nouvelle de l'existence du fac-similé des Noces de Cana, plusieurs Vénitiens s'imaginaient une surface luisante comme celle d'une affiche, et ils étaient terrorisés en pensant que cela constituerait la réparation qui leur était due après le viol culturel commis par Napoléon à San Giorgio. Ils ne pouvaient pas savoir que le fac-similé était composé de pigments imprimés sur une toile recouverte de plâtre tout à fait «semblable» à celle employée par Véronèse. Lorsque l'œuvre a été dévoilée, il y eut un moment de silence; ensuite des applaudissements enthousiastes et plusieurs larmes. Bon nombre de Vénitiens ont eu à se poser une question difficile : comment est-il possible d'avoir une expérience esthétique et une réaction émotive devant une copie? Cette question a ensuite donné lieu à celle-ci : comment peut-on empêcher que Venise soit inondée de mauvaises copies si nous ne comprenons pas les critères permettant de distinguer les bonnes des mauvaises transformations? 
Encore une fois, des techniques numériques nous permettent de distinguer des caractéristiques que les chercheurs ont trop rapidement inscrites sous la rubrique générique de la «reproduction». Comme nous l'avons constaté, le même élan de simplification intellectuelle était en jeu et les mêmes erreurs catégorielles ont été commises lorsque Benjamin a écrit son texte sur la «reproduction mécanisée ». Certes, il s'agit d'une question de précision, de compréhension et de respect - et l'absence de ces facteurs produit des répliques «serviles». Les mêmes techniques de reproduction peuvent être employées de façon servile ou de façon originale. Tout dépend des aspects que l'on choisit de mettre en relief et de ceux que l'on met hors jeu. Il se pourrait que le choix d'une technique s'inspirant de photographies, dans laquelle le restaurateur applique d'infimes points au lieu d'appliquer la peinture par traits plus larges, semblables à ceux de l'artiste, permette au restaurateur de mieux contrôler et dissimuler ses interventions. Mais cela ne fait que prouver qu'une reproduction manuelle est infiniment plus subjective et contestable qu'une reproduction «mécanisée ». L'enfer est pavé de bonnes intentions.

Sans doute le combat sera-t-il difficile: les fac-similés jouissent d'une mauvaise réputation - les gens assimilent ces derniers à une retranscription photographique de l'original - et le numérique est associé à une surenchère de virtualité. Dès lors, en parlant de «fac-similés numériques », nous semons très certainement le désordre. Et pourtant, nous soutenons, à l'encontre de ces préjugés assez répandus, que les fac-similés numériques sont en train de transformer la trajectoire séculaire des œuvres d'art. Il n'y a rien de particulièrement «virtuel » dans les techniques numériques - par ailleurs, il n'y a rien d'entièrement numérique dans les ordinateurs numériques ${ }^{6}$ ! Lassociation du numérique et du virtuel est le résultat de mauvaises habitudes dues à l'utilisation de seulement l'un des terminaux possibles de nos ordinateurs: celle de nos écrans qui restent de fort piètre qualité. Il en va autrement lorsque les techniques numériques ne constituent qu'un moment dans le processus menant d'une entité matérielle la version n-1 des Noces de Cana de Véronèse au Louvre - à une autre entité tout aussi matérielle - la version $n+1$ à San Giorgio. À l'époque du tourisme de masse et des campagnes de rapatriement des biens spoliés pendant des guerres ou

6. Voir Adam Lowe et Simon Schaffer, $n 01$ se, 2000 (exposition tenue simultanément au Kettle's Yard, Cambridge, au Whipple Museum of the History of Science, Cambridge, au Museum of Archaeology and Anthropology, Cambridge, et au Wellcome Institute, Londres). Voir également Brian Cantwell Smith, «Digital Abstraction and Concrete Reality», dans Impressiones, Madrid, Calcografia Nacional, 2003. 
soustraits dans le cadre d'échanges commerciaux, campagnes qui se font entendre de façon de plus en plus insistante; à l'époque où les restaurations relèvent de l'iconoclasme et où le nombre délirant d'amateurs risque de détruire même les œuvres les plus résistantes conservées dans les meilleures institutions, il devrait aller de soi que les fac-similés numériques nous proposent un nouvel outil tout à fait remarquable pour révéler la notion d'originalité que requiert cette nouvelle ère. Dans la mesure où tous les originaux devront être reproduits de toute façon, ne serait-ce que pour survivre, il importe plus que jamais de distinguer les bonnes des mauvaises reproductions.

Traduit de l'anglais par Eduardo Ralickas 
